\title{
ИСПОЛЬЗОВАНИЯ POWЕR FX ДЛЯ РАБОТЫ В COLLABORATION PLATFORMS
}

\section{USING POWER FX FOR COLLABORATION PLATFORMS}

\section{Voronkov S. Saradgishvili}

Summary. This article is devoted to an example of organizing work on building ETL processes in an enterprise contour. This technique can serve as an illustration of the idea of two key trends in the development of IT technologies: the simultaneous growth of opportunities that are provided by certain software products in conjunction with a decrease in the entry threshold. The review is carried out on the Microsoft 365 platform. Separately, we note that this language is part of the Power Platform. This is a relatively new vision of Microsoft corporation about the business data warehouse as a single point of connection and storage of data, tools. Together with the Power platform authors researched the Power automate product - a workflow designer, partly the successor to SSIS, SharePoint workflow engine. These tools can automate enterprise activities. To enhance the ability to develop with third-party technologies and programming languages, Microsoft has introduced the Microsoft Graph API.

Keywords: ETL, Collaboration Platforms.

\author{
Воронков Илья Александрович \\ Аспирант, Санкт-Петербургский политехнический \\ университет Петра Великого \\ iliaftk@outlook.com \\ Сараджишвили Сергей Эрикович \\ К.т.н., дочент, Санкт-Петербургский \\ политехнический университет Петра Великого \\ SSaradg@yandex.ru
}

Аннотация. Данная статья посвящена примеру организации работы по выстраиванию ETL-процессов в контуре предприятия. Данная методика может служить иллюстрацией идеи о двух ключевых тенденциях в развитии ИТ-технологии: одновременный рост возможностей, которые предоставляют те или иные программные продукты в совокупности со снижением порога входа. 06зор выполнен на решении платформе Microsoft 365 как наиболее часто встречающегося решения по созданию Collaboration platform в учреждениях за рубежом. Отдельно отметим, что данный язык является частью Power platform. Это относительно новое видение корпорации Microsoft 0 бизнес-хранилище данных как 0 единой точке подключения и хранения данных, инструментов. Вместе с Power platform свое развитие демонстрирует продукт Power automate — конструктор рабочих процессов, отчасти наследником SSIS, SharePoint workflow engine. Вместе эти инструменты способны закрыть большинство задач по автоматизации деятельности предприятия. Для расширения возможности разработки с использованием сторонних технологий и языков программирования Microsoft представила Microsoft Graph API.

Ключевые слова: ETL, платформы для совместной работы.

С точки зрения бизнеса, данная парадигма - это наиболее эффективный способ решения задачи, так как бизнес-пользователи привыкли мыслить именно задачами, которые должна решать та или иная система. К сожалению, на данном этапе развития технологий мы не в состоянии полностью перейти на подобные технологические решения. Причины могут быть разными: невозможность полностью решить задачу, используя лишь средства конструктора, нехватка специалистов, обладающих навыками моделирования, программирования и бизнес-опытом, наличие большой прослойки legacy code. Тем не менее стоит отметить рост подобных решений не только в сфере бизнеса, но и в научной среде [3-4]. Одновременно с развитием технологий все острее становится вопрос о месте данных решений в будущем. Многие владельцы крупных IT-компаний продвигают идею того, что программирование и разработка софта должно перестать быть узкоспециализированной областью [5]. Основная мысль сводится к тому, 


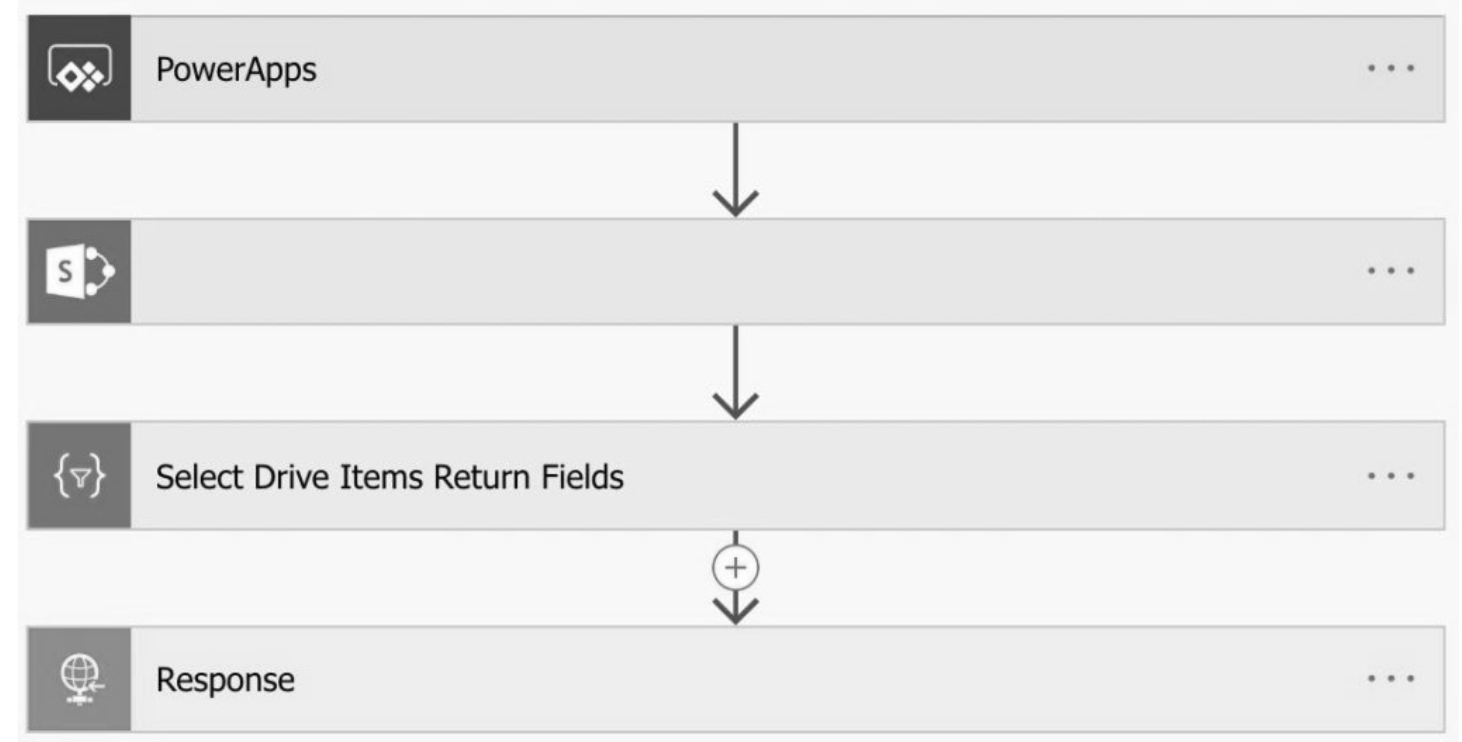

Рис. 1. Общая схема Flow

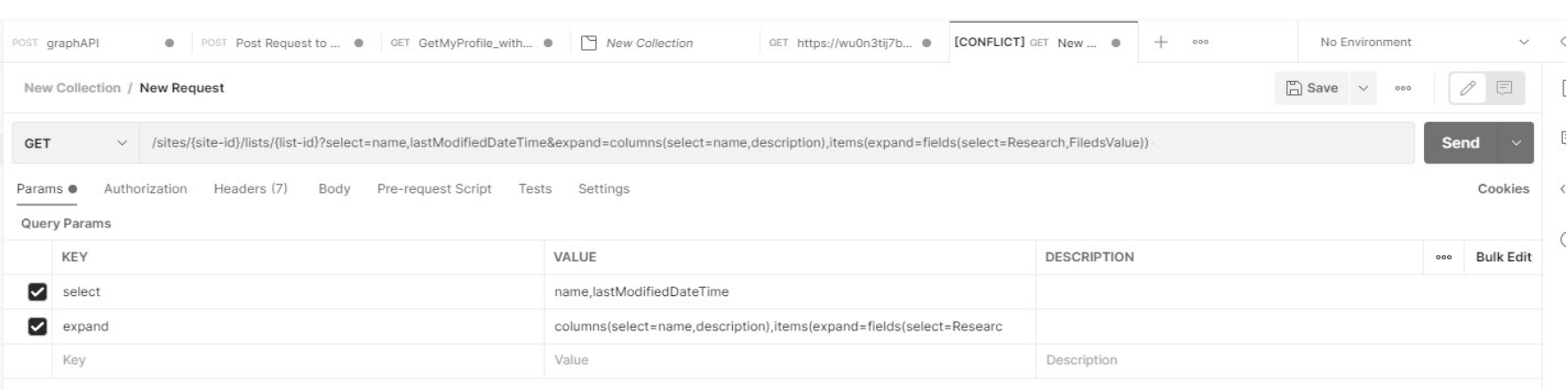

Рис. 2. Схема GET запроса

что в будущем люди будут решать с помощью программных инструментов свои персонализированные задачи. Для этого вам не придется получать образование, проходить специальные курсы. Все, что вам следует знать, это то, чего вы хотите добиться с помощью программы, и как она может облегчить вам жизнь. Конечно, подобные идеи могут казаться утопическими и несбыточными на горизонте ближайших 10-20 лет, но, оглядываясь на историю развития ИТ-отрасли мы начинаем думать, что все это может произойти уже в ближайшем будущем [6]. Рассмотрим задачу по настройки ETL процесса из корпоративного хранилища данных. Существует несколько подходов по организации работы подобного рода[7]. Большинство из них сводятся к серьезным вложениям средств на этапе проектирования, разработки, приемке. В данной работе используется механизм Lowcode работы, который становится все более популярным за счет низкой стоимости создания, но высокой операционной стоимости эксплуатации[8][9]. В обзоре представлен механизм получения элементов из произвольного хранилища, роль которого выполняет SharePoint. Адрес хранилища (сайт коллекция) может динамически изменяться, получая новый URL в качестве входного параметра. Задача состоит в универсальном подходе получения как данных из источника (бинарные файлы), так и извлечению информации метаданных. 


\begin{tabular}{|c|c|c|c|}
\hline \multicolumn{3}{|c|}{ Get Drive } & $\cdots$ \\
\hline${ }^{*}$ Site Address & & $x$ \\
\hline *Method & \multicolumn{2}{|l|}{ GET } & $\checkmark$ \\
\hline${ }^{*}$ Uri & \multicolumn{2}{|c|}{$f_{x} \quad$ If $(\ldots) \times$} & \\
\hline \multirow[t]{2}{*}{ Headers } & Accept & application/json & \multirow[t]{2}{*}{$x$ : 团 } \\
\hline & Enter key & Enter value & \\
\hline Body & \multicolumn{2}{|c|}{ Enter request content in JSON } & \\
\hline
\end{tabular}

Pис. 3. Динамическое подключение к SharePoint

$\{\nabla\}$ Select Drive Items Return Fields

$$
\begin{aligned}
& \text { *From } \\
& \text { *Map }
\end{aligned}
$$

\begin{tabular}{|c|c|c|c|}
\hline Name & $\{0\}$ & name $x$ & X 通 \\
\hline CreatedDateTime & $\{\theta\}$ & createdDateTime $\times$ & X \\
\hline LastModifiedDateTime & $\{t\}$ & lastModifiedDa... $\times$ & $\times$ \\
\hline CreatedBy & (c) & createdBy $\times$ & $x$ \\
\hline LastModifiedBy & $\{(-)$ & lastModifiedBy $x$ & $\times$ \\
\hline PhotoFacet & (b) & photo $x$ & $\times$ \\
\hline WebUrl & (b) & webUrl $x$ & $\times$ \\
\hline DownloadUrl & $\{f\}$ & @content.dow... $\times$ & $\times$ \\
\hline Thumbnail & $f_{x}$ & if $(\ldots) \times$ & $x$ \\
\hline OdataNextLink & 5. & @odata.nextLink $x$ & $\times$ \\
\hline ParentReference & (c) & parentReference $x$ & $\times$ \\
\hline ContentType & (1) & listItem.conten... $\times$ & $\times$ \\
\hline Folder & $\{b\}$ & folder $x$ & $x$ \\
\hline Version & (b) & listItem.fields.... $\times$ & $\times$ \\
\hline DriveId & (b) & parentReferen... $\times$ & $\times$ \\
\hline Size & $\{c\}$ & size $x$ & $\times$ \\
\hline Fields & 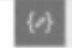 & listItem.fields $\times$ & $\times$ \\
\hline FieldsArray & $f_{x}$ & split(...) $\times$ & $x$ \\
\hline SiteId & $\{c\}$ & listItem.parent... $\times$ & $\times$ \\
\hline ListId & $\{a\}$ & listItem.parent... $\times$ & $\times$ \\
\hline Id & $\{a\}$ & id $x$ & $\times$ \\
\hline Enter key & \multicolumn{2}{|c|}{ Enter value } & \\
\hline
\end{tabular}

(a) value $x$


Таблица 1. Описание полей

\begin{tabular}{|c|c|c|}
\hline Свойство & Функция & Описание \\
\hline OdataNextLink & body('Get_Drive_Items')?['@odata.nextLink'] & $\begin{array}{l}\text { Добавление } \\
\text { автоматической } \\
\text { пагинации страниц }\end{array}$ \\
\hline DownloadUrl & item()?['@content.downloadUrl'] & $\begin{array}{l}\text { Это свойство } \\
\text { предоставляет } \\
\text { (временный) URL-адрес } \\
\text { с предварительной } \\
\text { аутентификацией } \\
\text { для каждого файла, } \\
\text { возвращаемого } \\
\text { в наборе результатов } \\
\text { выполнения Flow. }\end{array}$ \\
\hline Thumbnail & if(empty(item()['thumbnails']),,' item()['thumbnails'][0]['large']['url']) & $\begin{array}{l}\text { Получение свойства } \\
\text { предпросмотра } \\
\text { документа }\end{array}$ \\
\hline FieldsArray & FieldsArray & $\begin{array}{l}\text { Динамический набор } \\
\text { полей метаданных }\end{array}$ \\
\hline
\end{tabular}

\begin{tabular}{|c|c|c|c|}
\hline AbsoluteUri & DisplayName & Id & Value \\
\hline \begin{tabular}{|l|}
$m$ \\
\end{tabular} & MIG_280x60-1.png & $\begin{array}{l}\% 252 f L i s t s \% 252 f B P \_n o m i n e e \% 252 f \text { Att } \\
\text { achments\%252f75\%252fMIG_280x60- } \\
\text { 1.png }\end{array}$ & 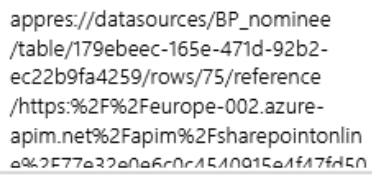 \\
\hline $\begin{array}{l}\text { https://thosharepoint.com } \\
\text { /sites/PowerAppsSandbox/Lists } \\
\text { /BP_nominee/Attachments } \\
\text { /75/sample.pdf }\end{array}$ & sample.pdf & $\begin{array}{l}\text { \%252fLists\%252fBP_nominee\%252fAtt } \\
\text { achments\%252f75\%252fsample.pdf }\end{array}$ & 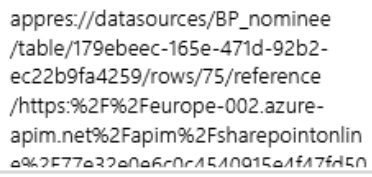 \\
\hline $\begin{array}{l}\text { https:/.sharepoint.com } \\
\text { /sites/PowerAppsSandbox/Lists } \\
\text { /BP_nominee/Attachments } \\
\text { /75/script.txt }\end{array}$ & script.txt & $\begin{array}{l}\text { \%252fLists\%252fBP_nominee\%252fAtt } \\
\text { achments\%252f75\%252fscript.txt }\end{array}$ & 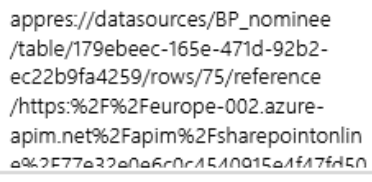 \\
\hline $\begin{array}{l}\text { https://,sharepoint.com } \\
\text { /sites/PowerAppsSandbox/Lists } \\
\text { /BP_nominee/Attachments } \\
\text { /75/Noronkov_Saeadzhashvilli.docx }\end{array}$ & Voronkov_Saeadzhashvilli.docx & $\begin{array}{l}\% 252 f L i s t 5 \% 252 \text { fBP_nominee\%252fAtt } \\
\text { achments } \% 252 f 75 \% 252 \text { fVoronkov_Sa } \\
\text { eadzhashvilli.docx }\end{array}$ & 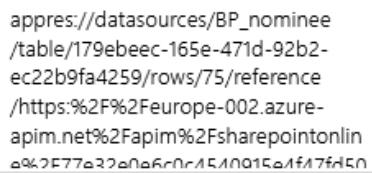 \\
\hline
\end{tabular}




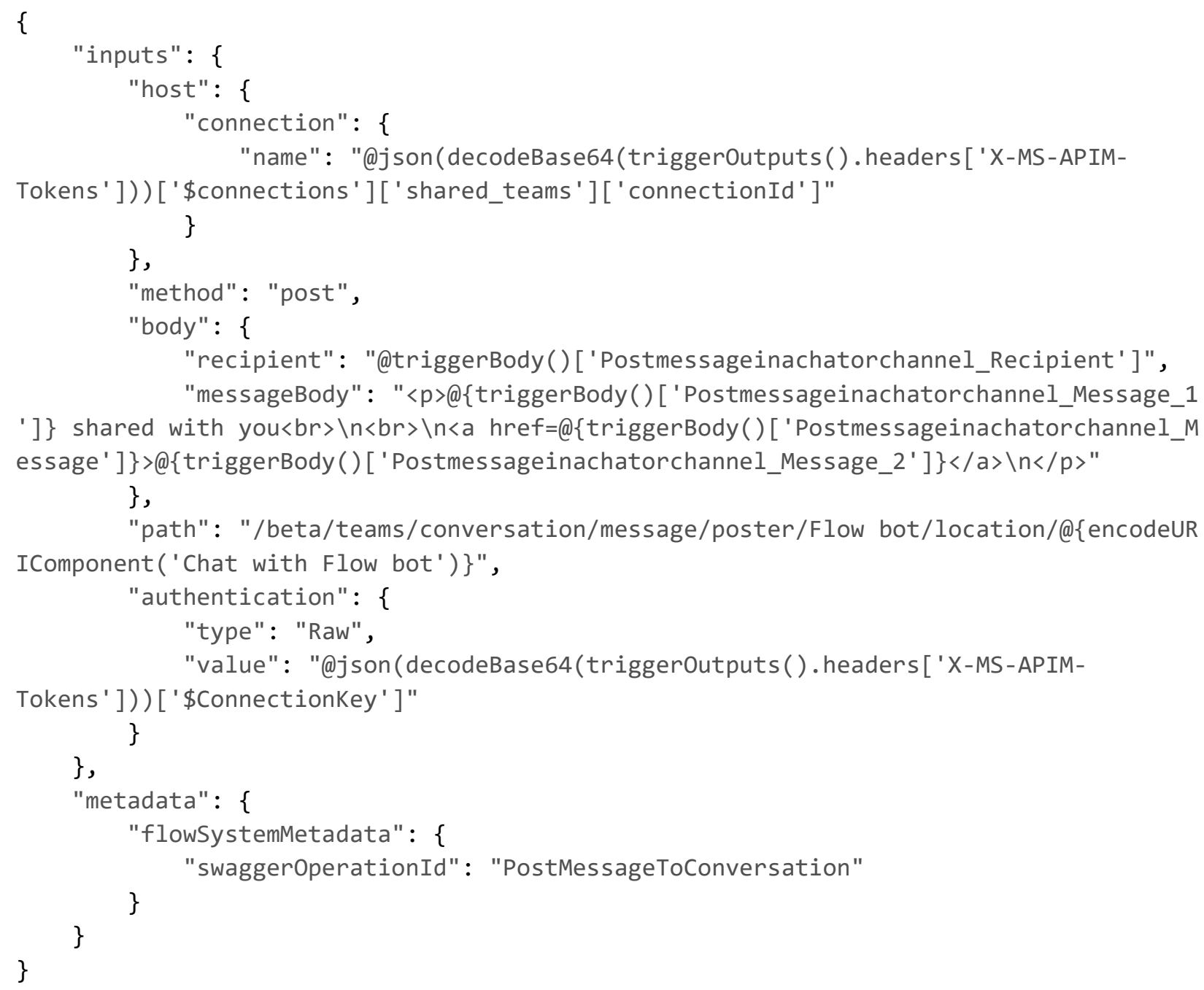

Рис. 6. JSON схема триггера

\section{Основная часть}

Приведем общую схему Flow получения динамических данных из хранилища SharePoint (рис. 1).

Семантику запроса можно обозначить в нотациях API (рис. 2).

Получение данных на уровне SharePoint можно задекларировать с наличием конструкции $\mathrm{IF}()$, которая будет меняться при вызове потока. Постоянным значением в данном блоке остается адрес тенанта, на котором развернут стенд (рис. 3).

Значение, переданное в этот входной параметр потока, игнорируется, если входной параметр odata.
nextLink, переданный в поток, имеет значение, отличное от false. Значение этого входного параметра должно быть в следующем формате: $\{$ siteld\}/lists/\{listld\}/root. Чтобы создать значение этого параметра для передачи в поток из PowerApps для любой заданной библиотеки документов на сайте, отображаемом в наборе результатов выполнения потока PA_Graph_Site_List, добавьте / root к значению SiteldListLibraryld, как определено на шаге «Выбор возвращаемых полей» указанного потока. Единственное отличие между получением списка файлов и папок из корневой папки библиотеки документов и получением списка файлов и папок с любой заданной папкой, содержащейся в библиотеке документов, заключается в том, что для корневой папки библиотеки документов необходимо изменить путь / root к значению SiteldListLibraryld, тогда как для папки 
вы вместо этого шага добавляется значение/ items / $\{$ itemld\}, где $\{$ itemld\} представляет этот идентификатор папки $[10,11]$. Идентификатор папки должен быть представлен в предыдущем прогоне потока того же потока, который определен на этапе выбора возвращаемых полей потока, когда запрашивались файлы и суппапки родительской папки текущего элемента. На шаге выбора возвращаемых полей в потоке значением поля Id будет \{itemld\}, а значением поля ContentTyре для этой строки будет Folder. Выборка полей метаданных для возвращаемых значений (рис. 4).

Наиболее значимые свойства полей, которые использует общее решение, представленное в этой статье, что, в свою очередь, позволило авторам реализовать полностью динамический облегченный пользовательский интерфейс, имитирующий подмножество функций, которые в противном случае были бы возможны только с использованием встроенного SharePoint включают (таб. 1):

Результат исполненного процесса можно изучить в low-code клиенте Power platform (рис. 5):

Данный пример ответа получен при выполнения операции получения поэлементно единицы массива из источника списка, где основной тип контента, кото- рый встречается на практике наиболее часто - это список (List ContetType). Список на платформе SharePoint или Dynamics 365 - это колоночное хранилище типизированных примитивов (текстовые строки, время, валюты, пользователи и другое), в которых по умолчанию нет возможности использования бинарных файлов, так как бинарные файлы как элементы решения располагаются в схожем типе хранилища данных, но имеют другой тип контента. Данная особенность связана с системой хранения информации на СУБД, где бинарный фал преобразуется в значение BLOB формата. В нашем примере мы использовали динамическое нахождение связи между двумя типами данных: реляционного представления метаинформации и бинарных файлов. Сложность этого шага связана с примером полезной нагрузки, которую авторы используют для создания схемы, необходимой для этого действия. Код такого взаимодействия через триггеры приведен ниже (рис. 6):

\section{Зак^ючение}

В данной статье приведена реализации организации ETL процесса по получению документов и метаданных с динамически изменяемым источником данных. Данный метод может быть использован для других источников данных, который поддерживают интеграцию с платформой Microsoft 365.

\section{ЛИТЕРАТУРА}

1. Rezaee Jordehi A. Optimal placement of battery swap stations in microgrids with micro pumped hydro storage systems, photovoltaic, wind and geothermal distributed generators/ A. Rezaee Jordehi, Mohammad Sadegh Javadi, João P.S. Catalão //International Journal of Electrical Power \& Energy Systems Volume 125, February 2021, 106483.

2. Gisbert R.S. Low-Code as Enabler of Digital Transformation in Manufacturing Industry/ R.S. Gisbert, 0. Garcia, F. Fraile // Appl. Sci. 2020, 10(1), 12; https://doi. org/10.3390/app10010012.

3. How M.L. Artificial Intelligence for Social Good in Responsible Global Citizenship Education: An Inclusive Democratized Low-Code Approach / M.L How, Y.J. Chan, S.M. Cheah, A.C. Khor // 19-21 of February 2021 in Vienna, Austria at the 2nd World Conference on Teaching and Education https://doi.org/10.6084/ m9.figshare.14038811.v1.

4. Rauschmayr N. Amazon SageMaker Debugger: A system for real-time insights into machine learning model training [Electronic resource] / N. Rauschmayr, V. Kumar, R. Huligol and others //Amazon Science — URL: https://www.amazon.science/publications/amazon-sagemaker-debugger-a-system-for-real-timeinsights-into-machine-learning-model-training (accessed:01.04.2021).

5. Straschnov E. You Shouldn't Have to Learn How to Code [Electronic resource] / E. Straschnov // Ве6-страница-URL: https://www.huffpost.com/entry/youshouldnt-have-to-lear_b_6111914 (accessed:01.04.2021).

6. Floridi L. GPT-3: Its Nature, Scope, Limits, and Consequences / L. Floridi, M. Chiriatti // Minds and Machines — 2020 — № 30. — P. 681-694.

7. Balasingham R. Designing a Data Warehouse System for Sales and Distribution Company / R. Balasingham, R. Subash // Big Data Mining and Analytics 2021 - № 1 (6).-P. $1-7$.

8. Dipanker J. Salesforce Integration Architecture/J.Dipanker, J. Hutcherson. - Apress, 2021. - 383 p.

9. Mccarthy S. Reusing Dynamic Data Marts for Query Management in an On-Demand ETL Architecture / S. Mccarthy // PhD thesis, Dublin City University. — 2021.

10. Voronkov I.A. Usage of collaborative filtering in sharing platforms / I.A. Voronkov, S.E. Saradgishvili //Journal of Physics: Conference Series — № 1679 (3). - P. 1-6.

11. Voronkov, I.A., Saradgishvili, S.E. Usage of a BART algorithm and cognitive services to research collaboration platforms // Journal of Physics: Conference Series, 2020, 1694(1), 012028. 